\title{
Diagnosing Bipolar Disorders in a Wearable Device
}

\author{
Chao Guil ${ }^{1}$, Jie Zhu ${ }^{2}$ \\ Department of Electronic Engineering \\ Shanghai Jiao Tong University \\ Shanghai, 200240, China \\ gl0901@126.com¹, zhujie@sjtu.edu.cn²
}

\begin{abstract}
Bipolar disorder, also known as a manic-depressive illness, is a common chronic recurrent psychosis and it mainly relies on doctors' experience to determine the patient's condition currently. Internationally, the doctor or mental health professional conduct a complete diagnostic evaluation by combining their auditory sense with YMRS (Young Mania Rating Scale). However, YMRS have significant limitations, e.g. extensive medical experience, Subjectivity, Misjudgment. For overcoming the traditional limitations, we aimed to find a useful methodology to diagnose the mental state and guide medical treatment by using speech signal processing.
\end{abstract}

The structure of the mania speech processing in this paper is depicted in Figure 1.

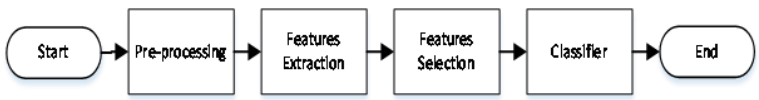

Figure 1. Structure of the Mania Speech Processing

The core issues of this paper are the feature extraction and classifier. In our research, we extract the six feature classes: pitch, formant, LPC, LSF, MFCC, PLPCC, GT filter. LPC is the most important feature of this article. In order to intuitively compare the assorted ability of different features, the features of Between-class Variance and Within-class Variance are calculated which shows in Figure 2.

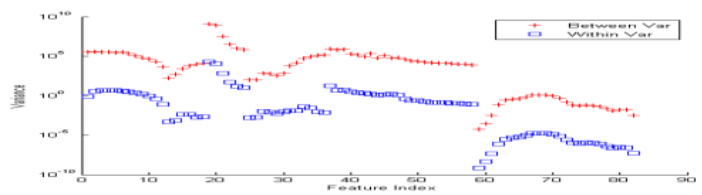

Figure 2. Feature assessment.

And then, we use SVM to classify the single patient and use GMM-UBM to classify multi patients whose processing could be seen in Figure 3.

In our system, we use genetic algorithm to select feature for SVM/GMM; and obtain optimal $\mathrm{c}$ and $\mathrm{g}$ for SVM. The classification accuracy is shown in Tables.
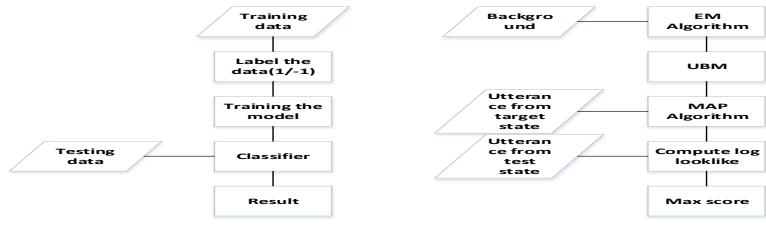

Figure 3. SVM/GMM processing.

Table 1. Result of Classification (N: Normal M: Manic)

\begin{tabular}{|c|c|c|c|c|c|c|c|}
\hline \multicolumn{3}{|c|}{$\begin{array}{c}\text { SVM for signal } \\
\text { patient }\end{array}$} & \multicolumn{3}{|c|}{$\begin{array}{l}\text { SVM for multi } \\
\text { patients }\end{array}$} & \multirow{2}{*}{\multicolumn{2}{|c|}{$\begin{array}{c}\text { GMM for } \\
\text { multi } \\
\text { patients }\end{array}$}} \\
\hline & $\mathrm{N}$ & $\mathrm{M}$ & & $\mathrm{N}$ & $\mathrm{M}$ & & \\
\hline $\mathrm{N}$ & 86.00 & 7.60 & $\mathrm{~N}$ & 58.53 & 39.42 & $\mathrm{~N}$ & 81.00 \\
\hline $\mathrm{M}$ & 14.00 & 92.40 & $\mathrm{M}$ & 41.47 & 60.58 & $\mathrm{M}$ & 72.00 \\
\hline
\end{tabular}

According to the experiment, we have found that the recognition rate of SVM classifier for a single patient were up to $90 \%$ and multi patients' rate of $50 \%$. The GMM classifier yields the better performance than SVM with a classification rate of $72 \%$.

The project's ultimate vision is develop a wearable device for diagnosis of affective disorders. As long as we set the parameters on the device in advance, patients could master their own mental state in real time by connecting the Bluetooth. As the Figure 4, we could get state when the speech data is upload to the Smart Bracelet.

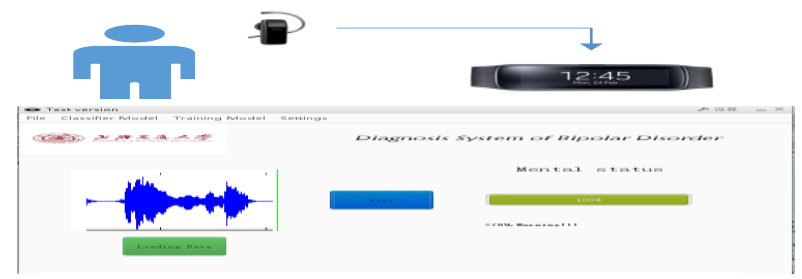

Figure 4. Wearable Device.

In our future works, the improvement of classifiers (SVM and GMM) will still be the core work in the next stage, and then, the all work will be fulfilled to provide a useful diagnostic tool for doctors.

\section{Keywords}

Bipolar Disorder, Support Vector Machine (SVM), Gaussian Mixture Model (GMM), Wearable Device.

This work was supported by the National Natural Science Foundation of China (NSFC) under Grants No. 61271349, 61371147and 11433002, the Shanghai Jiao Tong University joint research fund for Biomedical Engineering under Grant No. YG2012ZD04. 